\title{
Perfuração Tardia de Ventrículo Direito em Portador de Cardioversor Desfibrilador Implantável
}

\author{
Delayed Right Ventricular Perforation in Patient with Implantable Cardioverter - Defibrillator
}

Alexsandro Alves Fagundes, Luiz Pereira de Magalhães, Jussara Pinheiro, Leonardo Flausino, Luciano Rapold Souza

Hospital Português da Bahia, Salvador, BA - Brasil

Descrevemos o caso de uma paciente de 62 anos que retornou para avaliação nove meses após implante de cardioversor desfibrilador implantável (CDI) com sinais de perfuração tardia do ventrículo direito. São discutidos os sinais clínicos que permitem o diagnóstico dessa apresentação tardia, assim como as condutas e a frequência dessa complicação na literatura.
We describe the case of a 62-year-old patient who returned for evaluation nine months after receiving an implantable cardioverter-defibrillator (ICD) with signs of delayed right ventricular (RV) perforation. The clinical signs that allowed the diagnosis of this late presentation to be achieved are discussed herein, as well as the conduct and the frequency of this complication in the literature.

\section{Introdução}

Complicações tardias podem ocorrer em portadores de Cardioversor Desfibrilador Implantável (CDI). Contudo, manifestações tardias de perfuração do miocárdio nem sempre são evidentes. Assim, sinais clínicos e eletrônicos característicos dessa complicação devem ser conhecidos para que possam ser pesquisados.

\section{Relato do caso}

Paciente do sexo feminino, 62 anos, foi submetida a implante de CDI unicameral modelo Atlas VR St Jude em fevereiro de 2008 por episódio de taquicardia ventricular sustentada com pré-síncope. Naquela ocasião, foi realizado um ecocardiograma que demonstrou fração de ejeção de $45 \%$, com pequeno aneurisma na porção basal do ventrículo esquerdo (VE). Uma cineangiocoronariografia foi realizada: coronárias isentas de aterosclerose. A sorologia para Chagas foi positiva.

A avaliação de 30 dias não demonstrou eventos arrítmicos. A impedância e o limiar de estimulação do eletrodo ventricular estavam normais. A paciente permaneceu assintomática, em uso de amiodarona, carvedilol e enalapril.

Após nove meses do implante, a paciente compareceu para a segunda avaliação a despeito de ter sido orientada a revisão com 90 dias. Nesse momento, queixava-se de "pancadas" na barriga, sem sensação de choque. À avaliação clínica, chamava a atenção a presença de soluços, compatível com estimulação

\section{Palavras-chave}

Desfibriladores implantáveis, ruptura do septo ventricular, eletrodos implantáveis.

Correspondência: Alexsandro Alves Fagundes •

Rua Prof. Amilcar Falcão, 20/901 - Barra - 40140-480 - Salvador, BA - Brasil E-mail: alexfagundes@cardiol.br, alexfagundes2@gmail.com

Artigo recebido em 17/06/09; revisado recebido em 16/08/09; aceito em 04/03/10. diafragmática. À interrogação eletrônica, evidenciou-se ausência de captura e sensibilidade ventricular. Havia vários episódios de detecção inadequada de taquicardia ventricular deflagrados por ruídos no canal de sensibilidade, tratados com estimulação antitaquicardia (ATP) (fig. 1). O diagnóstico diferencial desse achado é o de um ruído de interferência externa (choque elétrico, interferência magnética) ou ruído interno por problemas no condutor do eletrodo. Nesse caso, os achados de perda de captura e sensibilidade ventricular afastaram a possibilidade de interferência externa. Tais dados levantaram a suspeita de fratura do eletrodo. A paciente foi orientada para internação de urgência.

A radiografia de tórax demonstrou a posição do eletrodo nitidamente fora da área cardíaca, encostado ao gradil costal. O ecocardiograma não demonstrou derrame pericárdico e a tomografia de tórax confirmou a presença da ponta do eletrodo na cavidade torácica, perfurando a parede livre do ventrículo direito (fig. 2).

Após discussão com a equipe da cirurgia cardíaca, optouse por remover o eletrodo sob visão direta com toracotomia, o que foi realizado sem intercorrência. Durante o ato cirúrgico, a remoção do eletrodo se deu sem dificuldades, e macroscopicamente não foram identificadas lesões no isolamento ou no condutor do eletrodo. Um novo eletrodo foi implantado por via endovascular sob visão fluoroscópica no septo ventricular. A paciente evolui sem intercorrências ou eventos arrítmicos nos últimos oito meses de seguimento.

\section{Discussão}

A perfuração aguda das câmaras direitas durante o implante endovascular de eletrodos é complicação pouco comum. Geralmente, a apresentação é catastrófica, com instabilidade hemodinâmica por tamponamento cardíaco e potencial desfecho fatal. Outro dado importante dessa complicação é que a manifestação geralmente é reconhecida na fase aguda peri-implante ${ }^{1}$. Sua identificação requer um alto grau de suspeição clínica diante de sinais de instabilidade hemodinâmica, aumento da silhueta cardíaca, estimulação 


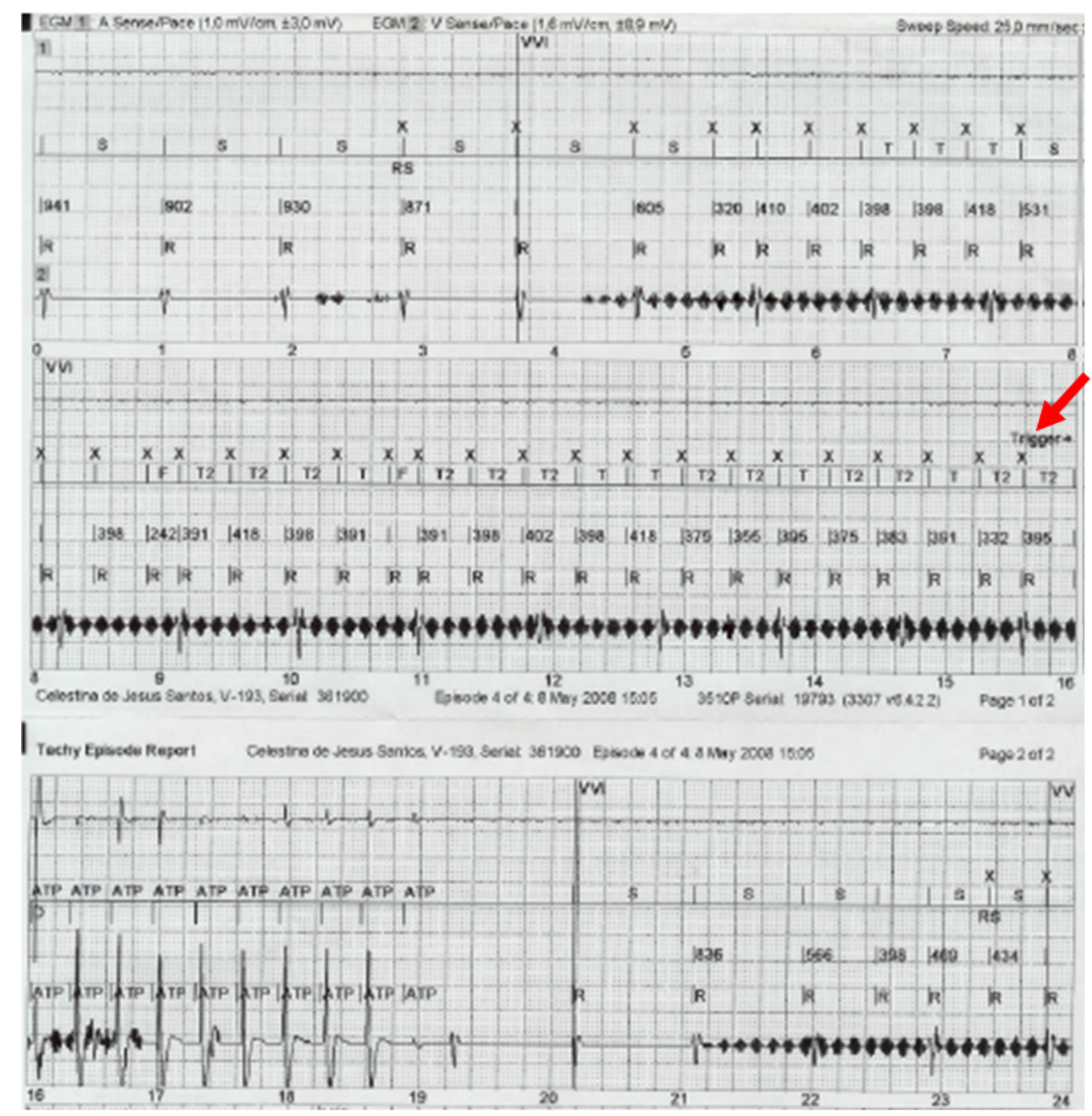

Fig. 1 - Terapia de ATP inapropriada por ruído. No traçado de baixo se observam artefatos de alta frequência. A contagem da frequência pelo CDI é determinada pelos eventos marcados com a letra R. Após 12 eventos (R) sentidos na zona de taquicardia (T2) uma terapia antitaquicardia (ATP) é iniciada (seta). Note que a interferência persiste mesmo após a terapia.

frênica e perda do eletrograma intracavitário. A radiografia de tórax e o ecocardiograma pós-implante podem ser úteis na identificação de apresentações subclínicas. Além das complicações hemodinâmicas potencialmente fatais, a perfuração do VD pode comprometer a função de reconhecimento de arritmias ventriculares, inviabilizando o potencial de proteção do CDI. Há relato de caso de perfuração tardia do VD identificada inicialmente pela perda do potencial intracavitário, ainda que sem modificação da posição do eletrodo à radiografia ${ }^{2}$.

Recentemente têm sido relatadas na literatura apresentações tardias de perfurações cardíacas não documentadas na fase aguda do implante. Em um estudo retrospectivo recente, 100 pacientes com marcapasso ou CDI implantado foram submetidos a tomografia de tórax. Todos os pacientes eram assintomáticos. Foram encontradas perfurações em até 15\% dessa casuística, incluindo perfurações atriais. O tempo médio entre o implante e a detecção radiológica da perfuração foi de 106 meses. Nos pacientes sem perfuração, o tempo médio entre o implante e a realização da TC de tórax foi de 43 meses $(p=0,0002)$. Isso demonstra como podem evoluir cronicamente perfurações não reconhecidas clinicamente. Fatores relacionados à cardiopatia estrutural, assim como o tipo de eletrodo utilizado podem estar relacionados a essa complicação. Todos os pacientes estavam com os dispositivos normofuncionantes, sem alterações de impedância, e não houve casos de tamponamento cardíaco. Os autores apontam para a natureza benigna e assintomática dessa complicação frequentemente não reconhecida e provavelmente muitas vezes sem necessidade de intervenção ${ }^{3}$.

O comprometimento da sensibilidade do eletrograma intracavitário compromete a detecção de arritmias ventriculares inviabilizando o potencial de terapia, assim como pode determinar ruídos no circuito de sensibilidade ventricular e deflagrar choques inapropriados. Nessa paciente, felizmente os ruídos deflagraram terapia indolor (ATP) que não foi percebida pela paciente.

Perfuração do VD é, portanto, complicação relativamente frequente e subdiagnosticada. Nem sempre o aspecto radiológico é diagnóstico. Alterações de sensibilidade e estimulação frênica devem ser valorizadas, o que foi decisivo para a suspeita clínica no nosso caso. A maioria 


\section{Relato de Caso}

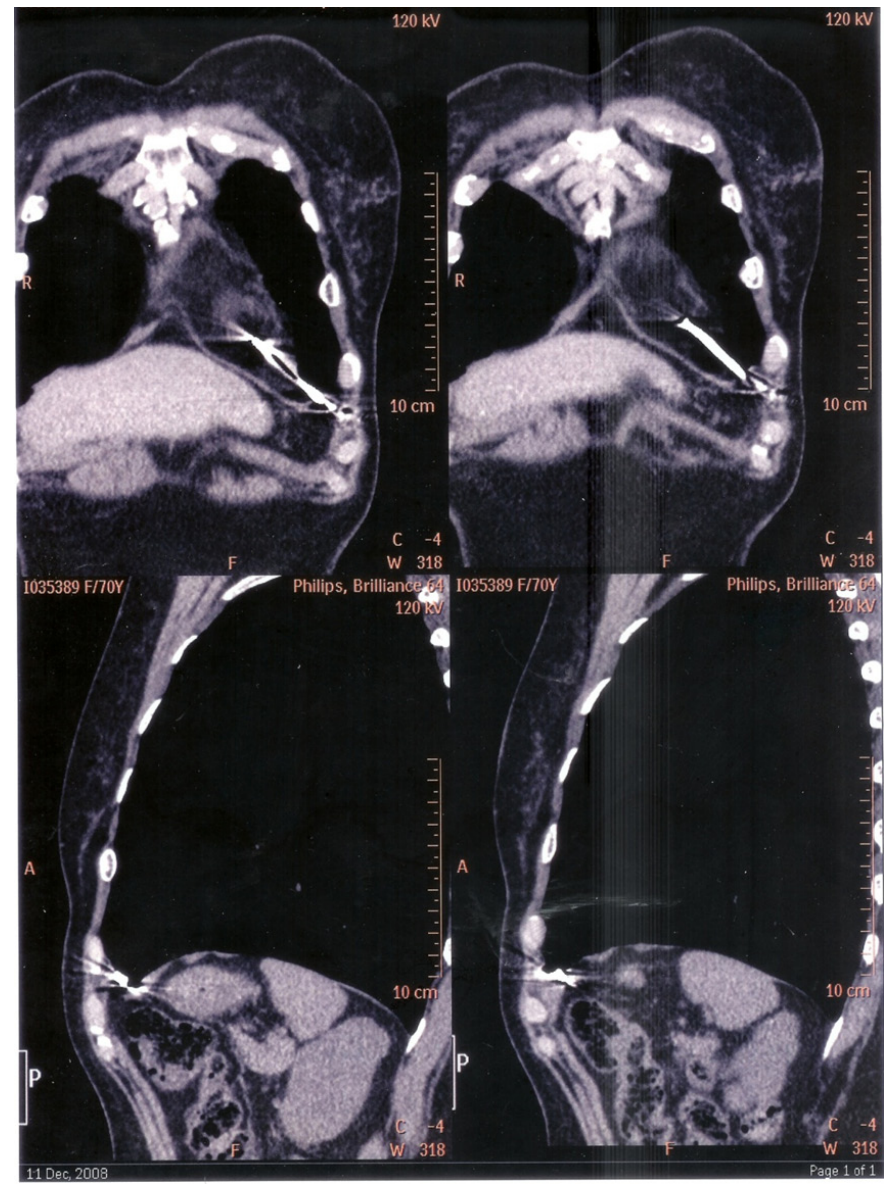

Fig. 2 - TC de tórax. Observe a ponta do eletrodo transfixando o VD e alojada no gradil costal.

das apresentações tardias tem evolução benigna; contudo é necessária uma atenção especial aos parâmetros de sensibilidade, impedância e limiares, que podem comprometer a função de terapias para arritmias ventriculares.

\section{Potencial Conflito de Interesses}

Declaro não haver conflito de interesses pertinentes.

\section{Fontes de Financiamento}

O presente estudo não teve fontes de financiamento externas.

\section{Vinculação Acadêmica}

Não há vinculação deste estudo a programas de pósgraduação.

\section{Referências}

1. Geyfman V, Storm RH, Lico SC, Oren JW 4th. Cardiac tamponade as complication of active-fixation atrial lead perforations: proposed mechanism and management algorithm. Pacing Clin Electrophysiol. 2007; 30 (4): 498-501.

2. Satpathy R, Hee T, Esterbrooks D, Mohiuddin S. Delayed defibrillator lead perforation: an increasing phenomenon. Pacing Clin Electrophysiol. 2007; 30: 28-32. Pacing Clin Electrophysiol. 2008; 31 (1): 10-2.

3. Hirschl DA, Jain VR, Spindola-Franco H, Gross JN, Haramati LB. Prevalence and characterization of asymptomatic pacemaker and ICD lead perforation on CT. Pacing Clin Electrophysiol. 2007; 30 (1): 28-32. 